\title{
DISCUSSION
}

\section{On the micromechanics of crushable aggregates}

\author{
G. R. M. McDOWELL and M. D. BOLTON (1998). Géotechnique 48, 667-679.
}

M. H. de Freitas and C. Butenuth, Imperial College of Science, Technology and Medicine, London

A. Khodaii, Amir Kabir University of Technology, Tehran

The authors have provided an intriguing insight into the possible development of fabrics arising from grain crushing. Such fabrics have long been recognized in geology where the term cataclasis (Gr. breaking) is used to describe their formation and the term cataclastic to describe their character. Rocks containing such fabrics are found in zones of concentrated shear displacement, and often contain fragments that have clearly been rolled. Weak sandstones exhibit similar features when sheared (Dobereiner \& de Freitas, 1986). Excellent descriptions of these rocks and of the history of their investigation are provided by Higgins (1971). Although such rocks were first recognized in the field over one hundred years ago (Lapworth, 1885), and have been much studied since, their formation remains a matter of curiosity. Experimental work recently reported by Renner \& Rummel (1996) and Menéndez et al. (1996) will acquaint the interested reader with modern views on the subject, and reveal the overlap which exists between the concepts used in structural geology and soil mechanics to explain both the formation and behaviour of cataclastic material.

In these studies, observers must decide on the circumstances which cause grains to fail in tension, and the authors rightly raise this issue in their discussion of fractal crushing. Briefly, the probability of small grains containing a large flaw is low, however the probability of them also having the lowest number of contact points (i.e. the smallest coordination number) is high. Thus, small grains may be intrinsically strong but have the greatest likelihood of carrying the highest stress. The authors describe succinctly the dilemma this causes: 'There are two opposing affects on particle survival, size and coordination number'.

Could it be that this dilemma is more apparent than real? Butenuth (1995) reviewed the results of tensile tests undertaken by others on single crystals, polycrystalline monomineralic rocks, polymineralic rocks and non-crystalline solids (glasses of various sorts), including the glass threads studied by Griffith and later by Gooding (1932). These all showed a loss in the magnitude of ultimate tensile stress they could sustain as the diameter of their sample increased; however, when the force required to fail the specimens is compared with the area of failure so formed, two apparently different types of relationship emerge - a straight line and a curve. The first may be a special case of the second, and this remains to be clarified, but it is evident that the straight-line relationships are frequently seen and appear to be constant over a wide range of areas in many types of material.

As an example of this, Fig. 10(a) illustrates the results obtained by Wijk et al. (1997) from carefully controlled tensile tests on solid cylinders of granite from Bohus in southern Sweden, and Fig. 10(b) the same material tested under a point load. Figs 10(c) and Fig. 10(d) illustrate both results recalculated as the mean force at failure $(F)$, calculated from the mean values of ultimate stress at failure indicated by Wijk, and area of failure $(A)$ (Butenuth, 1997). If a linear relationship between the points is used, the ratio $(\Delta F / \Delta A)=8.009 \mathrm{MN} / \mathrm{m}^{2}$ $+1 \times 10^{-3}$ with a correlation of 0.996 , and $8.0897 \mathrm{MN} / \mathrm{m}^{2}+$ $2.2 \times 10^{-4}$ with a correlation of 0.991 . Although the meaning of the intercept remains uncertain, two interesting trends emerge; (a) the slope $(\Delta F / \Delta A)$, which can be considered a measure of tensile strength, is for practical purposes the same in both cases, and (b) this result has been obtained from quite different shapes and sizes of sample. Butenuth (1997) gives other examples which show the same effect.

Applying this approach to the results of Lee (1992) produces the relationships shown in Fig. 2. To obtain these data the values for mean tensile strength recorded by Lee (1992) and reproduced in Fig. 12 by the authors, were compared with the average particle size to which they are attributed, using the same relationship employed by Lee, and based on the work of Jaeger (1967), and Shipway \& Hutchings (1993), namely $\left(\sigma=F d^{-2}\right)$, where $F$ is a maximum diametral force applied to a particle of diameter $d$ just before catastrophic failure occurs and the particle splits. Fig. 2 does not indicate a serious loss in the slope at which the force of failure increases to match the failure area it produces. Although the slope in each case is different, the slope of the force required to fail the grains appear close to constant over one order of magnitude change in area of failure for Leighton Buzzard sand, over two orders of magnitude for carboniferous limestone and over three orders of magnitude for oolitic limestone.

Are the size and shape controlling the micromechanics of failure in the way traditional thinking suggests? It is difficult to find evidence from cataclastic fabrics which clearly shows there is a preference for particles of one size to fail before another; the drawings of particle fracture in Dobereiner \& de Freitas (1986) illustrate typical occurrences. Perhaps greater progress would be made in studying this subject if some attention were directed to the force applied and the area of failure it creates in addition to the force applied and the area of contact over which it is presumed to operate. One interpretation of Fig. 2 is that it may be unsafe to claim anything more at present than that a particle of any size will fail when the force upon it is sufficient!

\section{Authors' reply}

We are very grateful to the authors of this discussion for their support regarding the direction of our research, and for the additional references they have provided. They raised the interesting question of the negative correlation between strength and sample size in brittle materials. In our paper we associated this phenomenon with plastic hardening in soils, arguing that irrecoverable volumetric compression was associated with the progressive crushing of the materials, and therefore with the progressive reduction in effective grain sizes. The discussers seem to be using their sources to make two different points:

(a) Tensile strength may be seen to vary with sample size not because of inherent strength loss in the material of the larger samples but because the proportional area of fast fracture changes as sample size increases.

(b) The nature of the sample size effect, however caused, can empirically be allowed for by fitting a straight line to the data of maximum fracture force plotted against gross area, and describing the slope of this line as the inherent strength.

Point $(a)$ can be seen as a micromechanical restatement of Weibull's postulate, as we now show. For approximately geometrically similar particles, we suggested that Weibull statistics can be applied to Jaeger's (1967) characteristic stress at failure $\sigma=F / d^{2}$. Such an approach predicts the average tensile strength to be given as $\sigma_{\text {av }} \propto d^{-3 / m}$, where $m$ is the Weibull modulus. For grains of soil of size $d$ suppose that the size of the critical flaw $a$ is given by $a \propto d^{x}$. For $x=1$, we have 


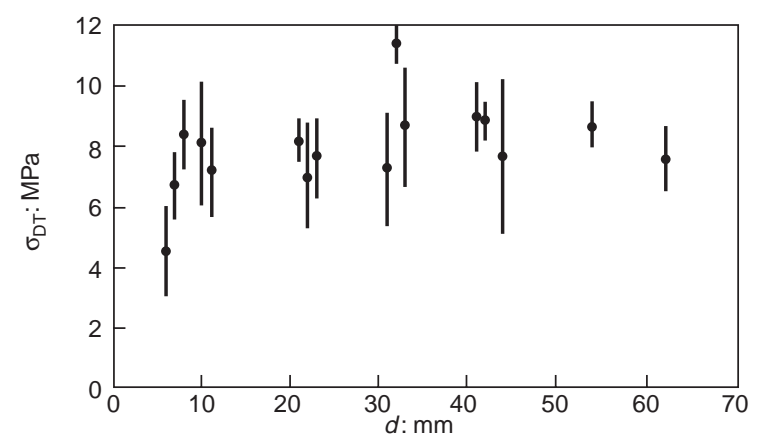

(a)

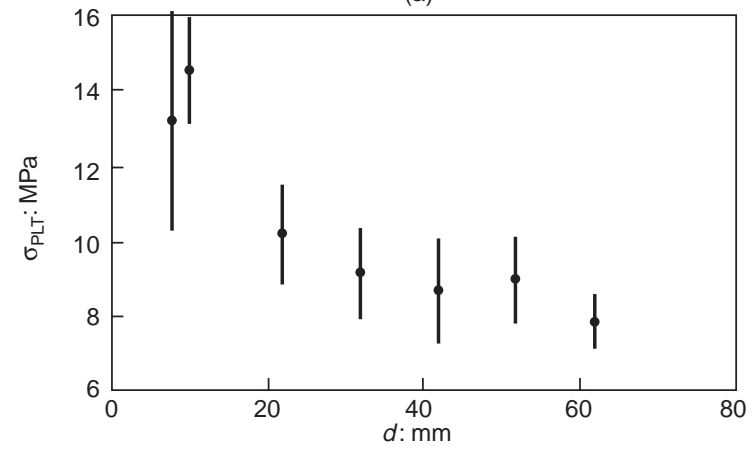

(b)

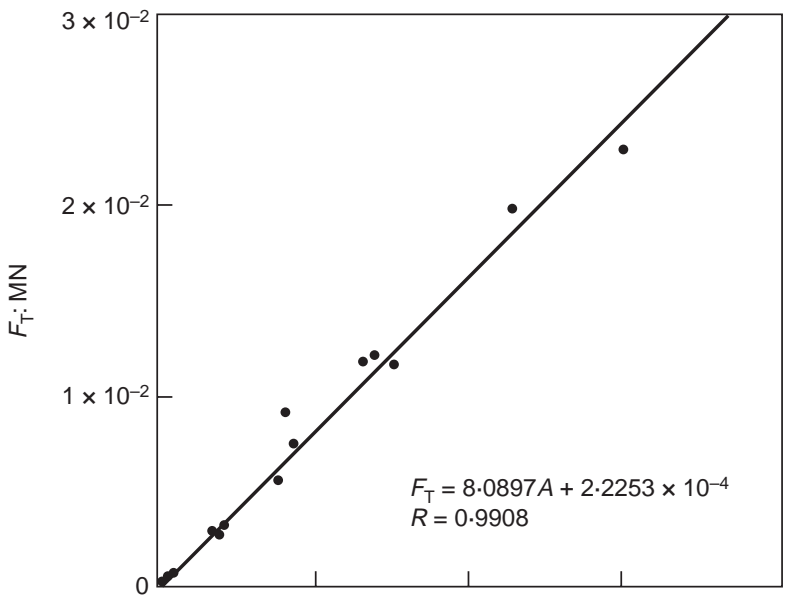

(c)

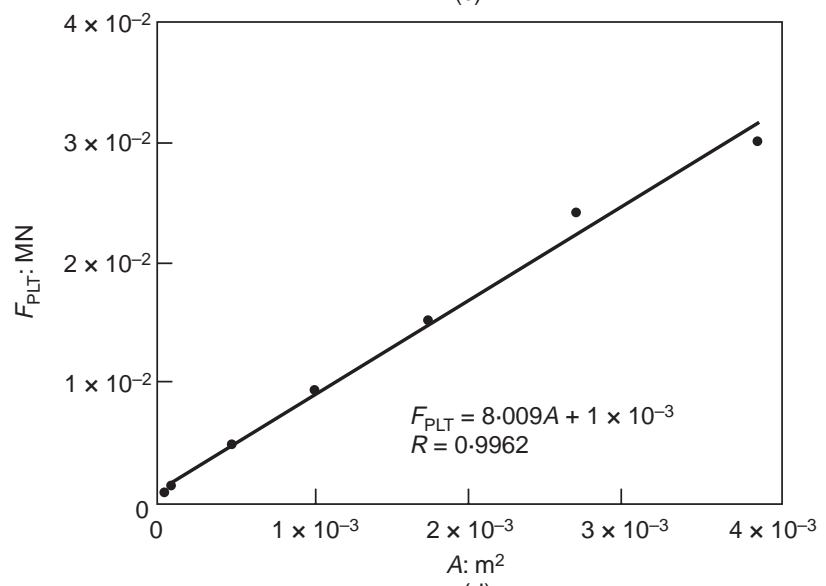

(d)

Fig. 10. Results from tensile tests on Bohus granite (see text)

Griffith's law for a disordered material $\sigma_{\mathrm{av}} \propto d^{-1 / 2}$, corresponding to a Weibull modulus of 6 . For $x<1$, as grain size decreases, the size of the critical flaw becomes a higher proportion of the size of the particle, representing a narrower distribution of flaws, less variability and $m>6$. Engineering ceramics have $m \approx 10$. A value of $x>1$ implies that as the particle size increases, the flaw size increases at a faster rate,
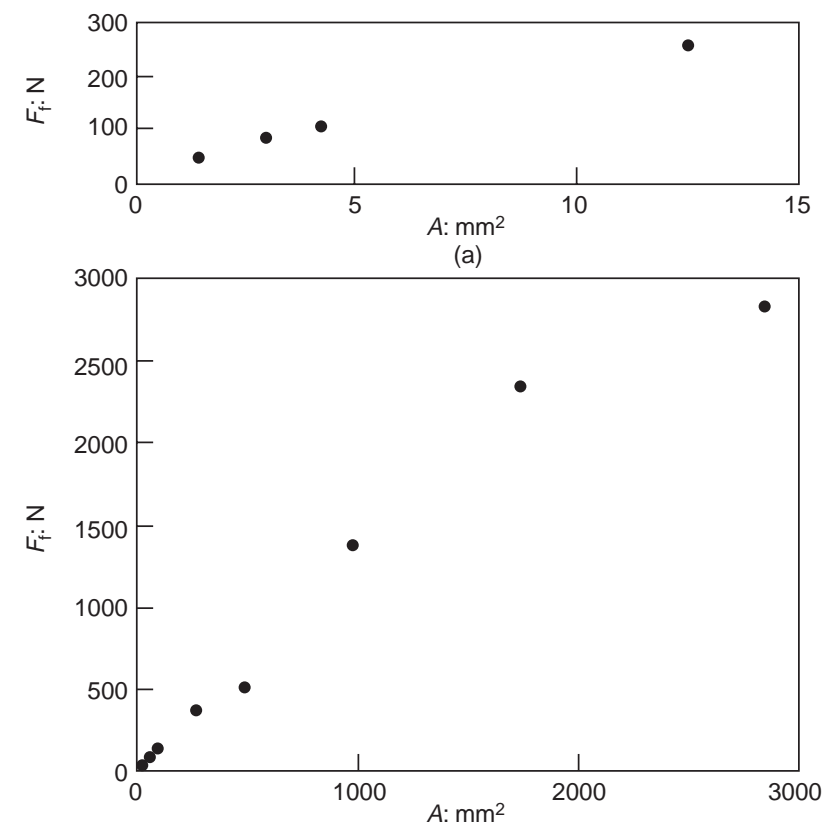

(b)

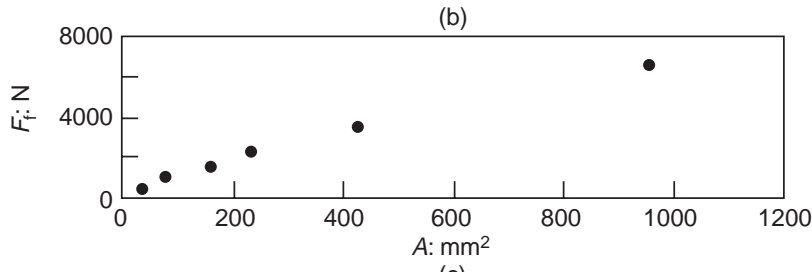

(c)

Fig. 11. Plots of results obtained by Lee (1992) recalculated as force at failure versus area of failure so caused. (a) Leighton Buzzard sand. (b) oolitic limestone. (c) carboniferous limestone

implying an upper limit to the possible size of particle. This corresponds with $m<6$.

For Lee's data (Lee, 1992), it was found that $\sigma_{\mathrm{av}} \propto d^{-b}$, and the parameter $b$ can be converted to a Weibull modulus $m$ for approximately geometrically similar grains using $m=3 / b$. The lowest Weibull modulus in Lee's data occurs for carboniferous limestone, with $m \approx 7$, implying a ceramic material of fair quality. The average crushing force is then given by $F_{\mathrm{av}}=$ $\sigma_{\text {av }} d^{2} \propto d^{2-b}$. If the new surface area $A$ produced at failure is taken to be proportional to $d^{2}$, then $F_{\text {ac }} \propto A^{1-b / 2}$. For the least ordered material in Lee's data, carboniferous limestone, $b=0.42$ giving $F_{\mathrm{av}} \propto A^{0.79}$. For the least variable material, oolitic limestone, $F_{\mathrm{av}} \propto A^{0.83}$. The plots produced by the discussers to support point $(b)$, are therefore seen to be power curves, although they can be approximated as linear over some range of grain sizes since the power exponent is quite close to (but not equal to) unity.

It seems to be common ground amongst all research workers on brittle materials, including those mentioned by the discussers, that the point load tests, which we treat as analogous to particle contact forces in an aggregate, give data with size effects as we assumed. Larger grains actually do fail at proportionally smaller stresses. If, as the discussers say, this is due to proportionally reduced fracture areas, this evidence supports our use of Weibull's statistics. This seems to us to point to the advisability of permitting the data to be fitted by power curves, as it so naturally appears to do.

Recently, work has been done on calcareous Quiou sand. Particles ranging in size from 1 to $16 \mathrm{~mm}$ in diameter were crushed between flat platens. For each size the distribution of strengths was Weibullian, and the average $m$ value was found to be about 1.5 . When the $37 \%$ strength $\sigma_{0}$ was plotted as a function of average particle size at failure on a $\log -\log$ scale, the value of $b$ corresponded to an $m$ value of 1.5 , with a correlation of 0.98 (Fig. 3). In this case, it would be anticipated 


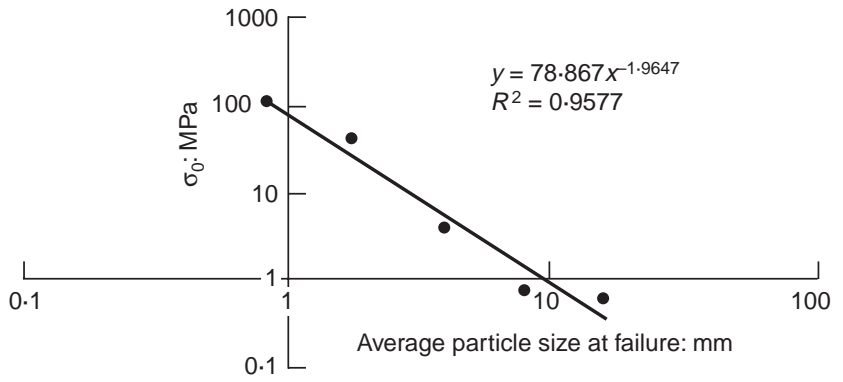

Fig. 12. $37 \%$ tensile strength as a function of average particle size at failure

that there would be no great variation in the average force measured at failure for the range of different particle sizes. It was found that there was no strong correlation between the average force at failure and average particle size at failure: the average crushing force varied from a minimum of $39 \mathrm{~N}$ to a maximum of $128 \mathrm{~N}$ for particles ranging in size from 1 to $16 \mathrm{~mm}$ in diameter. The average force at failure is plotted in the discussers' fashion as a function of $d^{2}$ in Fig. 13. The ratio of the maximum average crushing force to the minimum average force for the Quiou sand can be seen to be about 4, and this can be attributed to the difference in the values of $m$ between grain sizes and the variability in the strength of the material in general.

If force at failure had been proportional to area (i.e. to $d^{2}$ ), it would have been expected that the crushing force might increase by a factor of about 250 over the range of increasing grain sizes. For a soil with grains in which the size of the critical flaw is proportional to the size of the grain $(m=6)$, it would be anticipated that the average force at failure for the $16 \mathrm{~mm}$ grains might be greater than that for the $1 \mathrm{~mm}$ grains by a factor of $16^{1 \cdot 5}=64$. Fig. 13 illustrates the fact that the average force at failure is not a strong function of particle size. The best-fit straight line in Fig. 12 would have a clear intercept (which the discussers' ignore), a small slope (implying low tensile strength according to the discussers' definition), and a very poor correlation coefficient. We feel that this interpretation would not be useful for this soil. It should be noted that if the average force at failure in Fig. 13 is divided by the average $d^{2}$ at failure, this corresponds approximately to the average characteristic stress at failure, which when plotted as a functional of $d$ on a $\log -\log$ scale, gives a value of $b$ of 1.93 with a correlation of 0.98 . It seems that for this material the best definition of strength is the $37 \%$ or average characteristic stress at failure, which is a strong function of particle size.

Of course, we do not discount the possibility that some micromechanical theory can validate the fitting of straight lines to some force/area databases. On the other hand, we presently prefer to plot $\log -\log$ axes at the outset to discover whether the relation is a power curve to which Weibullian statistics would apply. However, we do strongly support the discussers' suggestion that more attention be directed to observing the geometry and mechanism of fracture produced by forces on grains.

We also believe that purely theoretical work is required to resolve the question of particle shape and relative size effects, raised by the discussers. In McDowell et al. (1996) we used a numerical program to study the effect of particle size and coordination number on the probability of fracture. We arbitrarily kept every particle under equal stress, so that contact force was effectively proportional to $d^{2}$. We assumed that smaller grains are stronger than larger grains. We then varied the effect of coordination number. For more angular grains, a high coordination number becomes less helpful. Similarly, for grains of a given shape and coordination number, the effect of the coordination number will change as the Weibull modulus changes. Our paper examined the relative influences of grain size and coordination number on the probability of fracture. If the effect of coordination number is removed, then the largest

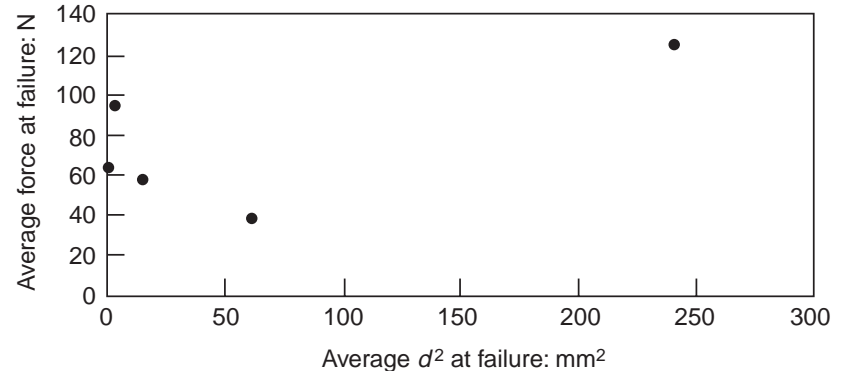

Fig. 13. Average force at failure as a function of average $d^{2}$ at failure

grains are always the weakest, so that a uniform matrix of very fine grains emerges. This sort of behaviour is not found in the soil mechanics literature. Usually, a proportion of the original grains remains, a distribution of grain sizes evolves, and the fine grains in the matrix soon represent a significant percentage by mass of the whole sample. Since mass is proportional to the $d^{3}$, then this means that most of the particles in the soil are very small. In that case, the small and medium-sized grains continue to disintegrate because of large tensile stresses induced in them by the few particle contacts. If coordination number is allowed to dominate, a fractal grain size distribution emerges.

Other statistical approaches are possible. It is evident from discrete element models of soil behaviour (Cundall \& Strack, 1979) that strong force chains are set up in granular aggregates, so that the stresses induced in small particles can be very large indeed. Consider grains compressed diametrically in a force chain. The strength of grains scales with size as $d^{-3 / m}$, but the stress induced in particles on a force chain scales as $d^{-2}$, which is much greater (for $m \gg 1 \cdot 5$ ). It seems that small grains are even more vulnerable than simple coordination concepts allow when contact forces are allowed to vary in accordance with statical principles. Nevertheless, the outcome should be the same-small grains fracture and particle sizes disperse as volume reduces due to improved packing. Stress rises as volume reduces because the smallest particles are getting smaller and proportionally stronger.

Future work must involve the numerical simulation of crushable aggregates under stress if a better understanding of the micromechanics of clastic soil is to be obtained. It may then be possible to define reliable soil parameters: those for which the physical origins are understood

\section{REFERENCES}

Butenuth, C. (1995). Gesteinfestigkeit und deren Veränderung als Grenzschichtproblem. Das Vorhaben wird durch das Ministerium für Wissenschaft und Forschung des Landes Nordhein-Westfalen, Bundersrepublik Deutschland, gefördert. Die Forderung erfolgt im Rahmen des Lise Meitner Programms.

Butenuth, C. (1997). Comparison of tensile strength values of rocks determined by point load and direct tension tests. Rock Mech. Rock Engng 30, 65-72.

Cundall, P. A. \& Strack, O. D. L. (1979). A discrete numerical model for granular assemblies. Géotechnique, 29, No. 1, 47-56.

Dobereiner, L. \& de Freitas, M. H. (1986). Geotechnical properties of weak sandstones. Géotechnique 36, 79-94.

Gooding, E. J. (1932). Investigation on the tensile strength of glass. $J$. Soc. Glass Tech. 16, 145-170.

Higgins, M. W. (1971). Cataclastic rocks. Geological Survey Professional Paper 689. Washington, DC: US Department of the Interior.

Jaeger, J. C. (1967). Failure of rocks under tensile conditions. Int. J. Rock Mech. Mineral Sci. 4, 219-227.

Lapworth, C. (1885). The Highland controversy in British geology; its causes, course, and consequences. Nature 32, 558-559.

Lee, D. M. (1992). The angles of friction of granular fills. $\mathrm{PhD}$ dissertation, University of Cambridge.

McDowell, G. R., Bolton, M. D. \& Robertson, D. (1996). The fractal crush-ing of granular materials. J. Mech. Phys. Solids. 44, No. 12, 2079-2102. 
Menéndez, B., Zhu, W. \& Wong, T.-F. (1996). Micromechanics of brittle faulting and cataclastic flow in Berea sandstone. J. Struct. Geol. 18, $1-16$.

Renner, J. \& Rummel, F. (1996). The effect of experimental and microstructural parameters on the transition from brittle failure to cataclastic flow of carbonate rocks. Tectonophysics, No. 258, 151-169.
Shipway, P. H. \& Hutchings (1993). Fracture of brittle spheres under compression and impact loading. I Elastic stress distributions. Phil. Mag. A 67, No. 6, 1389-1404.

Wijk, G., Rehbinder, G. \& Lögdström, G. (1997). The relation between uniaxial tensile strength and the sample size for Bohus granite. Rock Mech. Rock Engng 10, 201-219. 